\title{
Ethos et hybridité dans le texte discursif du roma La terre et le sang de Mouloud Feraoun.
}

\author{
Rania Hassan Ahmed \\ Professeur adjoint de linguistique à l'université de Hélouân, \\ Le Caire, L'Egypte. \\ Raniah279@gmail.com
}

\section{Résumé:}

Le présent article vise à étudier les stratégies discursives du texte énonciatif du roman La terre et le sang en vue de tracer les traits de l'éthos hybride dans le texte fictionnel de l'écrivain algérien d'expression française Mouloud Feraoun. Notre recherche tend à définir les articulations du moi à travers l'étude de la modalisation dans le texte énonciatif du roman de Feraoun. L'étude cherche également à interroger la dialectique de la personne dans le texte discursif du roman en le soumettant à une analyse quantitative et à une étude de la fréquence en vue de mettre en relief la subjectivité du texte de Feraoun.

Mots clés : Ethos, hybridité, identité, personne, modalisation, fréquence

الإيتوس و الهجنة في النص الخطابي من خلال رواية 》 الأرض و الدم 《 للكاتب مولود فرعون 


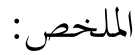

تمدف هذه الورقة البحثية إلى دراسة استراتيجيات الخطاب للنص التلفظي لرواية

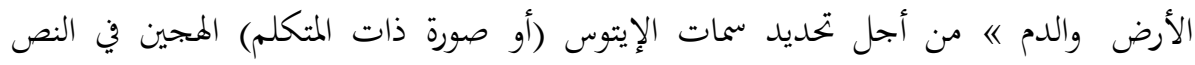

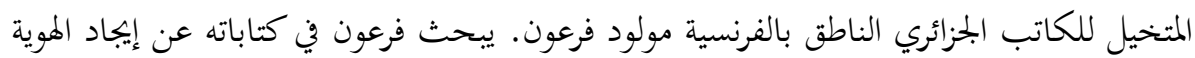
والاختلاف في نفس الوقت. ويهدف بحثنا إلى تحديد معالم و سمات ذات المتكلم من خلال دراسة مصوغات وموجهات المنطوق أو la modalisation في النص التلفظي لرواية فرعون. ونسعي من خلال دراسة موجهات النفي والتوكيد في النص الخطابي لرواية فرعون إلي تحديد

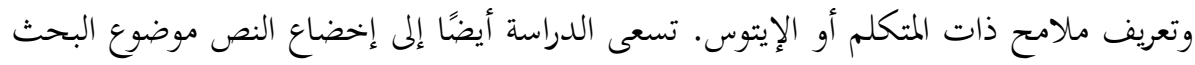

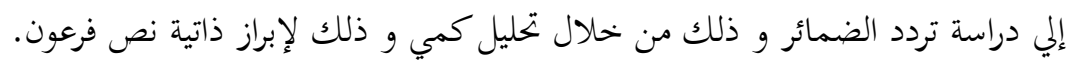
الكلمات المفاتيح: الإيتوس، الهجنة، الهوية، الضمائر، مصوغات المنطو ، التردد

Ethos and hybridity in the discursive text of the novel La terre et le sang of Mouloud Feraoun.

Abstract:

The present article aims to study the discourse strategies of the enunciative text of the novel La terre et le sang in order to trace the features of the hybrid ethos in the fictional text of the French-speaking Algerian writer Mouloud Feraoun. Our research tends to define the articulations of the ego through the study of modalization in the enunciative text of Feraoun's novel. The study also seeks to question the dialectic of the person in the discursive text of the novel by subjecting it to a quantitative analysis and a study of frequency in order to highlight the subjectivity of the text of Feraoun.

Key words: ethos, hybridity, identity, pronouns, modalization, frequency 


\section{Introduction:}

Le sens habite véritablement le texte comme une mystérieuse substance ; il est le fond de cette curieuse entité appelée forme et l'acte de lecture consiste à le dévoiler. ${ }^{(1)}$

La présente étude où nous adoptons le point de vue de l'analyse du discours, tient à dégager les stratégies discursives, les règles, les mécanismes et les outils langagiers dont l'auteur s'est servi en vue de projeter de lui-même une image de soi dans le texte discursif de son récit. Elle vise à tracer les articulations de l'ethos montré, selon les termes de Dominique Maingueneau, dans le texte énonciatif du récit fictionnel La terre et le sang de l'écrivain algérien d'expression française Mouloud Feraoun.

L'univers de sens que délivre le discours s'impose par l'ethos comme par les «idées » qu'il transmet ; en fait, ces idées se présentent à travers une manière de dire qui renvoie à une manière d'être, à la participation imaginaire à un vécu. Le texte n'est pas destiné à être contemplé, il est énonciation tendue vers un co-énonciateur qu'il faut mobiliser, faire d'adhérer «physiquement » à un certain univers de sens. ${ }^{(2)}$

Feraoun qui écrit en français incarne ainsi l'ethos de l'auteur algérien confronté à l'occidentalisation, à la francisation. $\mathrm{La}$ terre et le sang est un roman où Feraoun chante son amour de la Kabylie de telle façon qu'il plonge parfois son texte dans le pittoresque.

Feraoun surgit au sein de son récit impersonnel à travers un énoncé qui dévoile son image de soi, l'ethos de Feraoun, 
le kabyle qui attaque le stéréotype de l'écrivain algérien acculturé et exilé dans la langue du colon. Il déclare son kabylisme en s'exprimant en français. Ruth Amossy parle de l'indexation de l'ethos à un stock d'images préexistantes et de représentations collectives figées qui nourrissent l'ethos individuel et lui donnent son poids ${ }^{(3)}$. La terre et le sang se dresse contre ce stock d'images préexistantes et de représentations collectives qui nourrissent l'ethos de l'écrivain algérien d'expression française. Ce que Feraoun propose dans son écriture c'est de retrouver à la fois l'identité et la différence. Il interroge les signes qui lui manifestent à leur tour sa propre identité. La terre et le sang est un ouvrage qui incarne la recherche identitaire d'un héros kabyle, Amer, ayant quitté son village pauvre pour un voyage vers le pays du colon La France, retourne de nouveau vers ses sources kabyles après quinze ans d'exil. Le roman de Feraoun retrace les traits d'un ethos hybride, celui du protagoniste du roman de Feraoun qui s’identifie de temps à autre à l'être empirique du discours. Notre recherche tend à définir l'articulation entre la façon dont l'énonciation produit des ethos discursifs construits d'une part par les personnages fictionnels du roman et d'autre part par l'image de l'auteur qui s'y projette.

La présentation de soi s'effectue à travers un discours désireux d'amener l'auditoire aux vues du locuteur par des moyens qui ne sont pas seulement ceux de l'empathie et l'identification aveugle: elle s'insère dans un ensemble de 
stratégies verbales où les éléments de l'ethos, du logos et du pathos s'imbriquent étroitement les uns dans les autres. ${ }^{(4)}$

Le texte discursif du roman peint différents ethos: L'ethos de l'exilé, l'ethos du kabyle éprouvant une nostalgie à sa terre, l'ethos de l'homme humilié par la situation morose de son peuple, l'ethos de l'étranger souffrant de l'absence du minimum de communication avec son entourage, l'ethos de l'autre arraché à sa terre modernisée et civilisée. Marie, l'épouse française d'Amer, incarne ce dernier ethos. Marie qui elle aussi n'a pas le sang purement français, incarne l'image de l'exilée. Elle quitte l'occident pour vivre au sein d'un peuple déphasé, marginalisé, loin de toute modernisation. L'abandon de sa patrie lui cause elle aussi une crise identitaire. Feraoun retrace à travers son roman les périls de l'assimilation, le sentiment de l'externalité et de l'exil du moi humain. Le dialogue d'Amer et Marie incarne cette lutte du moi en vue d'une adaptation à l'univers de l'autre. En quittant son village kabyle, Amer a décidé de n'y plus revenir. Quand même il prend la décision d'y retourner, car « (il) sait c'est que d'être étranger, c'est un état pitoyable pour l'homme " ${ }^{(5)}$

Marie ressent cette étrangeté au sein de la famille d'Amer dont elle ignore tout : langue, mœurs et coutumes. Elle avoue à Amer qu'elle se sent plus ignorante que les femmes kabyles : « $(\ldots)$ depuis que je suis ici, je me sens un peu comme vos femmes : elles sont ignorantes, mais $j$ 'en sais encore moins qu'elles. $»^{(6)}$. À travers ce dialogue entre les 
deux moi hybrides _ où Le locuteur L se fait une image de lui-même et de son partenaire de la communication, de même, l'interlocuteur se fait une image de lui-même et de son locuteur _ plusieurs ethos nous sont révélés. Amer et Marie est un couple qui représente l'échec de l'acte de compréhension entre le moi et l'autre, cet échec symbolise la distanciation créée par le colonisateur français entre lui-même et le peuple algérien, échec symbolisé par l'histoire d'amour d'Amer et de Chahba la femme de son oncle Slimane. La présente recherche se charge alors d'étudier la modalisation et la personne dans trois extraits du texte discursif du roman $\mathrm{La}$ terre et le sang* en vue de détecter les traits de l'ethos de l'auteur s'articulant à travers les ethos de ses héros surtout celui d'Amer le protagoniste du roman. Notre étude est sensée répondre aux questions suivantes : Comment est-ce que l'auteur s'est forgé une image de soi et s'est construit une certaine identité à travers la manière de dire, la parole des héros du roman ? Comment est-il présent dans son récit impersonnel ? Et quelle stratégie a-t-il adoptée en vue de subjectiviser son roman?

2. La modalisation et l'adhésion du locuteur à son discours :

Dans la problématique de l'énonciation (acte de production du texte par le sujet parlant), la modalisation définit la marque donnée par le sujet à son énoncé, c'est la composante du procès d'énonciation permettant d'estimer le degré d'adhésion du locuteur à son énoncé. 
La modalisation est la prise en charge par le locuteur de son énoncé, elle est la marque de la présence du locuteur dans son énoncé d'où la part de la subjectivité qu'elle représente dans le texte discursif. La personne (je, nous,...) n'est pas la seule preuve de la présence du locuteur dans son discours, la modalisation indique le parti pris par le locuteur à l'égard de ce qu'il dit. Elle lui permet de s'impliquer ou de nuancer ce qu'il dit, d'y ajouter un commentaire. Elle est comme un dédoublement énonciatif qui permet au locuteur d'ajouter un commentaire réflexif sur son énoncé. Selon Todorov et Ducrot, il est à distinguer dans un acte d'énonciation un contenu représentatif, appelé dictum qui est la mise en rapport du prédicat avec son sujet, et une attitude prise par le locuteur à l'égard de ce contenu ou le modus. La modalisation comprend donc tous les indices qui marquent la présence du locuteur dans son énoncé. Elle est la preuve de sa présence et sert à indiquer son caractère. La modalisation en déterminant les termes de la relation du locuteur à son discours, sert à mettre en relief les traits de son ethos.

En soumettant notre corpus discursif à une analyse quantitative visant à repérer les taux de fréquence des modalisateurs dans le contexte dialogique, nous avons découvert que la catégorie des modalisateurs de négation et celle d'intensité sont considérées comme étant significatives car la fréquence de leur apparition est nettement supérieure à la moyenne. 
Tableau 1 : l'occurrence des modalisateurs

\begin{tabular}{|l|l|l|l|l|}
\hline \multirow{2}{*}{$\begin{array}{l}\text { Text } \\
\text { e }\end{array}$} & \multicolumn{2}{|l|}{ Pourcentage } & \multicolumn{2}{c|}{$\begin{array}{l}\text { Nombre } \\
\text { d'occurrence }\end{array}$} \\
\cline { 2 - 5 } & $\begin{array}{l}\text { Modali } \\
\text { s. } \\
\text { Négati } \\
\text { on }\end{array}$ & $\begin{array}{l}\text { Modalis. } \\
\text { Intensité }\end{array}$ & $\begin{array}{l}\text { Modalis. } \\
\text { Négation }\end{array}$ & $\begin{array}{l}\text { Modalis. } \\
\text { Intensité }\end{array}$ \\
\hline $\begin{array}{l}\text { Text } \\
\text { e 1 }\end{array}$ & $30.2 \%$ & $32.1 \%$ & 16 & 17 \\
\hline $\begin{array}{l}\text { Text } \\
\text { e 2 }\end{array}$ & $26.9 \%$ & $26.9 \%$ & 14 & 14 \\
\hline $\begin{array}{l}\text { Text } \\
\text { e 3 }\end{array}$ & $33.8 \%$ & $31.7 \%$ & 48 & 45 \\
\hline
\end{tabular}

Figure 1 : La fréquence des modalisateurs dans le texte 1

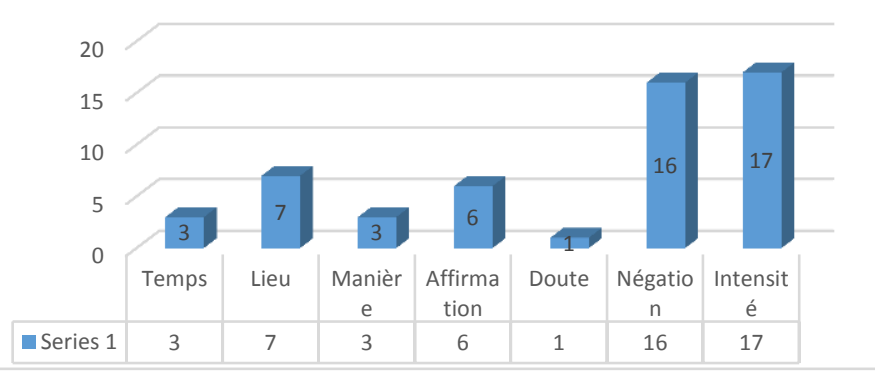


Figure 2 : La fréquence des modalisateurs dans le texte 2

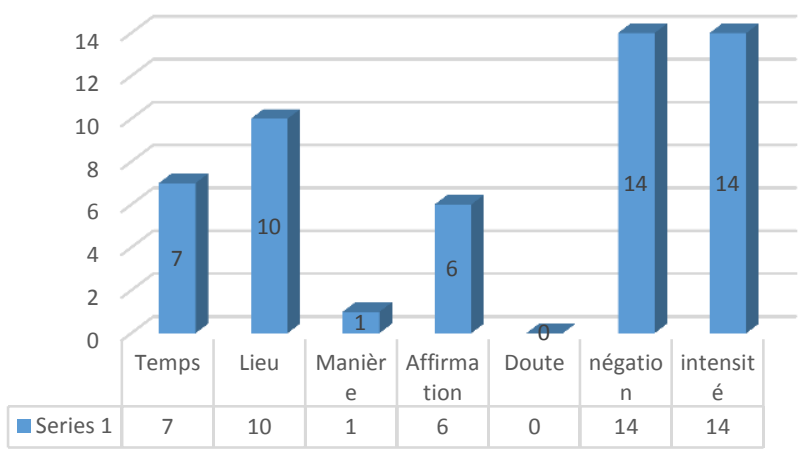

Figure 3 : La fréquence des modalisateurs dans le texte 3

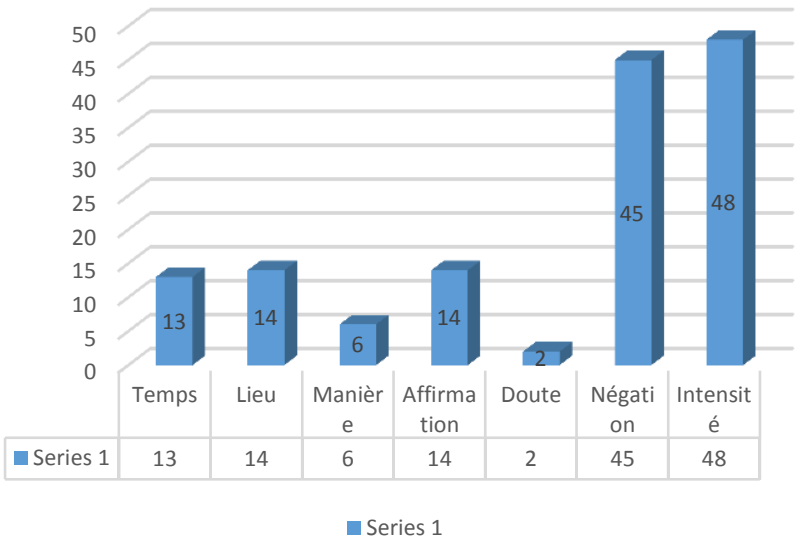

2.1. La négation, expression du rejet et de la dislocation du moi :

Les modalisateurs de la négation et de l'intensité servent à dramatiser le discours. Commençons par la négation qui est une stratégie discursive du rejet. Oswald Ducrot définit la négation comme le choc de deux attitudes opposées à l'intérieur d'un même énoncé produit par un même locuteur. 
La plupart des énoncés négatifs [...] font apparaître leur énonciation comme le choc de deux attitudes antagonistes, l'une, positive, imputée à un énonciateur E1, l'autre, qui est un refus de la première, imputée à E2. ${ }^{(7)}$

Il distingue deux types de négation : la négation descriptive et la négation polémique. La négation descriptive est de nature phrastique, elle est l'affirmation d'un contenu négatif et elle a affaire au contenu vériconditionnel de la proposition.

(La négation descriptive) sert à représenter un état de choses, sans que son auteur présente sa parole comme s'opposant à un discours adverse. ${ }^{(8)}$

Quant à la négation polémique, elle est de nature polyphonique, elle instaure une opposition qui n'est pas entre deux locuteurs mais entre deux points de vue de deux énonciateurs (E1) et (E2) d'un locuteur (L) dans l'énoncé (9). (L) met en scène (E1) l'énonciateur d'un énoncé effectif antérieur auquel s'oppose (E2) de l'énoncé négatif et réplicatif. (E2) rejette le point de vue de (E1) dans l'énoncé de (L). C'est ainsi que se traduit la dimension dialogique et polyphonique de la négation polémique.

Tout énoncé négatif n'est pas la réfutation d'un dire, ni d'une pensée, mais tout énoncé négatif convoque, fictivement, un dialogue polémique. ${ }^{(10)}$

Tableau 2 : Exemples de négations descriptive et polémique dans texte 1

Négation descriptive

Négation polémique 
1 Amer : Il ne s'agit pas Marie: Ce n'est pas pour te de cela le rappeler que j'en parle. Au contraire, je me trouve si heureuse depuis une semaine !

2 Amer : elle n'était pas Amer : elle n'est plus de notre avis étrangère. Elle entre dans la famille.

3 Amer: Ce rachat ne lui Marie: ne nous faisons pas dit rien trop d'idées. Nous vivrons simplement et nous serons ici plus tranquilles qu'ailleurs

4 Marie : je n’y comprends rien.

5 Marie : Plus de contremaître, ni de chef d'équipe

Tableau 3 : Exemples de Négations descriptive et polémique dans texte 2

La négation descriptive La négation polémique

1 Chahba : Vous n'avez pas Kamouma : je ne les ai pas gênés là, dans ma assez de place, ici soupente. Je tombe endormie chaque soir comme une souche

2 Chahba: Ce n'est pas bien Chahba : Il ne faut pas désespérer. Amer 
loin aura sa part.

3 Kamouma : il ne m'a jamais aimée.

4 Kamouma : Je n'y comprends rien.

5 Kamouma : Je ne dis pas non

Tableau 4 : Exemples de négations descriptive et polémique dans texte 3

\begin{tabular}{|c|c|c|}
\hline & La négation descriptive & $\begin{array}{l}\text { La négation } \\
\text { polémique }\end{array}$ \\
\hline 1 & $\begin{array}{l}\text { Ramdan : Je ne sais pas lire, } \\
\text { moi. }\end{array}$ & $\begin{array}{l}\text { Ramdan : Le vrai } \\
\text { village, ce n'est pas } \\
\text { celui qui se dresse } \\
\text { fièrement sur la crête. } \\
\text { C'est celui-ci : figé } \\
\text { dans notre terre, } \\
\text { immobile et éternel } \\
\text { mais peu effrayant à } \\
\text { mon avis }\end{array}$ \\
\hline 2 & $\begin{array}{l}\text { Amer: Mais je ne pourrais pas } \\
\text { les distinguer toutes. }\end{array}$ & $\begin{array}{l}\text { Ramdan : Oh ! Amer, } \\
\text { notre terre n'est pas } \\
\text { méchante. Nous en } \\
\text { sortons et nous y } \\
\text { retournons }\end{array}$ \\
\hline
\end{tabular}

3 Ramdan : nous n'avons pas 
peur d'y venir

4 Ramdan : Ce n'est pas pour te mettre en colère que je te rappelle ces choses

Le couple Amer et Marie incarne un exemple représentatif du «vivre-ensemble», deux cultures différentes se marient pour réaliser le rêve de Feraoun, ce rêve auquel Emmanuel Roblès fait allusion dans la préface du roman où il cite les paroles de Feraoun dévoilant sa colère contre ce divorce brutal entre La France et L'Algérie.

J'ai pour la Kabylie, écrivait (Feraoun), une tendresse filiale que j'ai voulu exprimer dans mes livres. J'en ai donné une image sympathique mais non une image trompeuse. (...) Il s'agit seulement de comprendre pourquoi cette unanimité dans la rébellion, pourquoi le divorce est brutal. La vérité, c'est qu'il n'y a jamais eu mariage ! (...) Ce qu'il eût fallu pour s'aimer? Se connaître d'abord. ${ }^{(11)}$

Le texte (1) de notre corpus discute cette question : comment comprendre l'autre, comment dissiper toute discordance et tout divorce entre le moi et l'autre. C'est ce que Marie et Amer essayent tous deux de faire. La négation joue un rôle important dans le texte discursif. Elle incarne d'une part la différence entre les deux mentalités, et d'une autre comment cette différence ne fait qu'unir les deux pôles du discours. Le texte est un échange entre les deux époux à propos de l'achat de Tighezzrane, cette propriété que la 
famille d'Amer a été obligé de vendre pour subvenir à ses besoins après le le départ du fils en France.

Récupérer Tighezzrane était un essai de la part du fils Amer de remédier au mal que sa migration a causé à sa famille. Tighezzrane est la terre- symbole de nostalgie et de retour aux origines. La négation est la dialectique de deux thèses antipodes : thèse proposée et thèse refutée. Ce dialogisme que créent les modalisateurs de la négation dans le texte (1) résume la relation entre ce couple Amer-Marie. Seize négations descriptives et trois polémiques sont repérées du texte 1 , donc seize négations touchant le contenu vériconditionnel (phrastique) de l'énoncé. La négation descriptive se sert d'une stratégie discursive qui représente des paroles négatives dont l'auteur ne se présente pas comme s'étant opposé à un discours antérieur. Ces types de négations ne nécessitent pas une correction comme son analogue polémique. Au contraire, dans la négation polémique 1, Marie nie cette idée de rappeler Amer de leur décision prise avant leur retour à Ighil-Nezman, et selon laquelle le couple retournerait à Paris une fois ça n'y marche pas. Marie se montre nier une thèse antérieure à son énoncé, et selon laquelle Amer voit que Marie lui rappelle de leur décision de retourner à Paris si les choses vont mal à Ighil-Nezman. Cela est clair par l'usage de cette structure binaire de la négation polémique où l'énoncé négatif «Ce n'est pas pour te le rappeler que j'en parle. » ${ }^{(12)}$ est suivi d'une correction : «Au contraire, je me trouve si heureuse depuis une semaine ! ${ }^{(13)}$. 
Nous remarquons alors ce dialogisme que crée la négation polémique entre thèse refutée (la thèse antérieure à l'énoncé) : Marie rappelle Amer qu'il s'agit de retourner chez elle une fois elle est repoussée par la famille de son époux; et la thèse proposée (la thèse présente dans l'énoncé) : Marie est heureuse à Ighil-Nezman avec la famille d'Amer et ne pense point à retourner chez elle.

Dans la négation polémique (2), Amer nie une thèse antérieure à son énoncé et selon laquelle : Une femme qui part et quitte son pays, même si elle était accompagnée par son homme, est malheureuse. Il corrige cette thèse réfutée dans son énoncé «elle n'est plus étrangère.» ${ }^{(14)}$ à l'aide d'un énoncé correctif «Elle entre dans la famille. » ${ }^{(15)}$.

Dans la négation polémique (3), Marie se montre nier une thèse opposée à son énoncé $E$ 《ne nous faisons pas trop d'idées $»^{(16)}$ et selon cette thèse antérieure, il s'agit de faire trop d'idées à propos de ce sujet du retour en France puisque Marie n'est pas à l'aise chez la famille de son époux. Marie l'énonciateur de $\mathrm{E}$ rectifie cette thèse fautive par un autre énoncé : «Nous vivrons simplement et nous serons ici plus tranquilles qu'ailleurs ${ }^{(17)}$.

Dans le texte (2) de notre corpus, cinq négations descriptives et deux négations polémiques sont repérées. Le dialogue est entre les trois héroïnes Chahba, Marie et Kamouma. Les trois femmes ont pour trait commun l'amour qu'elles portent envers Amer. Chahba est l'amante qui trouve dans son amour pour Amer une aventure qui mérite tout 
sacrifice. Amer est dans les yeux de Chahba l'homme qui a côtoyé l'occident et s'est ouvert à la culture de l'autre. Il differe de son mari Slimane plus âgé et ayant un esprit plus borné. L'arrivée de ce dernier a mis fin à la rupture entre les deux familles kabyles : les Ait-Larbi et les Ait-Hamouche. Chahba invite Kamouma à passer la nuit chez elle, cela ne lui était pas permis il y a des années à cause de la rupture entre les deux familles. Marie, ou « Madame » telle qu'elle est désignée par le narrateur dans le récit de Feraoun, ne saisit pas la plupart des échanges entre Chahba et Kamouma car elle ne maîtrise pas encore la langue kabyle. C'est pourquoi la part du lion du discours est résevée aux deux hérö̈nes kabyles qui se comprennent et s'entendent bien. Chahba s'efforce de gagner Kamouma dans sa part, elle l'invite chez elle pour dormir et laisser le couple jouir d'une nuit privée, c'est ce qui pousse Kamouma à rectifier l'erreur de la considérer comme étant un obstacle se dressant contre le bonheur de son fils en le privant d'être à l'aise avec sa femme. Le malice de Chahba est bien évidente. Kamouma rectifie ceci à l'aide d'une négation polémique : «je ne les ai pas gênés là, dans ma soupente. Je tombe endormie chaque soir comme une souche ${ }^{(18)}$. L'énoncé négatif traduit ce dialogisme polémique, l'énonciateur $\mathrm{E}$ nie un énoncé antérieur d'un énonciateur E1 selon lequel la présence de kamouma nuit à la vie privée des deux époux. La rectification de ce point de vue suit l'énoncé négatif : au contraire, Kamouma dort comme 
une souche donc, elle ne nuit point par sa présence au bonheur de son fils et son épouse.

Dans la négation polémique (2), Chahba ayant remarqué le désespoir aux yeux de kamouma, c'est le désespoir qu'Amer n'ait pas d'enfant, se précipite de nier cette idée et de la rectifier à l'aide de la structure binaire de la négation polémique :

Enoncé négatif + rectificateur

Il ne faut pas désespérer + Amer aura sa part.

Dans le texte 3 de notre corpus, ce texte qui s'étale sur six pages, un long discours entre Ramdan _le père de Chahba_et Amer, on a repéré 30 énoncés négatifs descriptifs et 2 énoncés négatifs polémiques. Ce discours est un essai de la part de Ramdan de reconcilier entre Amer et son oncle Slimane. Dans ce long discours qui a lieu au cimetière d'Ighil Nezman, Ramdan s'attarde à relater l'histoire des différentes familles du village, et comment les tombes sont disposées par karouba _par famille _ exactement comme au village. Ramdan présente à Amer le triste sort d'un étranger enseveli et enterré au cimetière du village. Mourir loin de sa terre est un sort horrible pour un kabyle. Ce sort a été subi par l'oncle d'Amer Rabah qui a été assassiné par le polonais André aux mines en France. Amer étant l'ultime témoin du meurtre ne voulait pas avoir affaire aux autorités françaises, vue la menace proférée par André de lui faire endosser le crime. La parole d'un immigré kabyle ne valait pas grand-chose face à celle d'un Européen. Au retour, Amer était accusé de trahison par 
Les Ait-Hamouche. Slimane le frère de Rabah était forcé par ses frères de se venger d'Amer. La négation dramatise la scène énonciative où Amer et Ramdan s'efforcent de nier l'un et l'autre tous les malentendus, les arrière-pensées et les fausses paroles qui se sont propagées dans le village et qui ont causé toute cette rancune entre l'oncle et le neveu. Amer nie sa culpabilité «Je sais que je ne suis pas coupable» et Ramdan le conseille de ne pas croire aux rumeurs qui se répandent au village «Il ne faudra pas écouter ces rumeurs. » ${ }^{(19)}$. La négation est atténué par l'usage des modalisateurs d'intensités : le verbe d'opinion savoir dans «je sais» et la forme impersonnelle d'obligation dans «il ne faudra pas». La modalisation d'intensité sert à dramatiser la scène d'énonciation et à influencer le lecteur en atténuant et en renforçant le degré de certitude d'une assertion dans «je sais que je ne suis pas coupable » et d'une obligation dans «Il ne faudra pas écouter ces rumeurs. ».

\subsection{L'intensité et la dramatisation du discours identitaire :}

On appelle modalisateurs les moyens par lesquels un locuteur manifeste la manière dont il envisage son propre énoncé ; par exemple, les adverbes peut-être, sans doute, les incises (à ce que je crois, selon moi,) etc., indiquent que l'énoncé n'est pas entièrement assumé ou que l'assertion est limitée à une certaine relation entre le sujet et son discours. ${ }^{(20)}$

Les modalisateurs d'intensité sont les moyens linguistiques à l'aide desquels le locuteur d'un énoncé amplifie ou adoucit le degré de puissance de son énoncé. Ils servent à marquer une 
certitude ou au contraire un degré d'incertitude du locuteur à l'égard du contenu propositionnel de son énoncé. Ils témoignent ainsi d'une forte implication du locuteur dans son énoncé. Cette variation de degré de puissance, de témoignage ou d'obligation d'un énoncé participe effectivement à la présentation de l'image de soi _ l'éthos _ dans le discours du locuteur.

Nous essayerons alors de déceler les traits de l'image de soi à travers le repérage des différents amplificateurs (les modalisateurs qui augmentent le degré d'intensité de l'énoncé) et adoucisseurs (les modalisateurs qui atténuent le degré d'intensité de l'énoncé) auxquels le sujet-parlant a recours en vue de marquer le degré de son adhésion à l'énoncé.

Le repérage des marques linguistiques de la modalisation d'intensité a été effectué grâce à une analyse quantitative.

Tableau 5 : Les modalisateurs d'intensité dans le texte 1

Enoncé

Modalisateurs

d'intensité

1 Amer : Plus de paie aussi ! Enfin Plus nous pourrons revendre, si un jour =amplificateur cela nous chante.

2 Marie : Veux-tu que je te dise? Un peu = Eh bien, moi, depuis que je suis ici, adoucisseur je me sens un peu comme vos femmes.

3 Marie : elles sont ignorantes, mais Encore moins 
j'en sais encore moins qu'elles = adoucisseur

4 Marie : En attendant de bien Bien= comprendre, je me laisse aller. amplificateur

Tableau 6 : Les modalisateurs d'intensité dans le texte 2 Énoncé Modalisateurs

d'intensité

1 Kamouma : Toi, ma fille, tu es plus Plus = difficile qu'on ne croit. amplificateur

2 Chahba : C'est ce qu'on va voir. Pas assez de= Vous n'avez pas assez de place, ici. atténuateur

3 Chahba :Tu viendras tous les soirs y Tous = dormir. amplificateur

4 Chahba: Ce n'est pas bien loin.

Bien amplificateur

Tableau 7 : Les modalisateurs d'intensité dans le texte 3

Énoncé

1 Ramdan : forcément. (Notre Forcément=amplificateur cimetière) s'agrandit petit à

petit, comme le village

2 Ramdan : Et puis aussi les Plus = amplificateur tombes se transforment. $\mathrm{Tu}$ vois? Toutes les nouvelles sont plus hautes. Certaines sont recouvertes de ciment.

3 Ramdan: Il y a même des Peut-être = atténuateur 
inscriptions ...en français. Des

noms. Et l'âge aussi, peut-être.

Je ne sais pas lire, moi.

4 Amer : $\mathrm{Tu}$ les connais toutes, je Toutes $=$ amplificateur parie.

Nous ne tenons pas à faire un inventaire exhaustif de marqueurs d'intensité, mais plutôt une exemplification de ces marqueurs (amplificateurs et atténuateurs) tels qu'ils sont observés dans le contexte de notre corpus discursif. L'emploi des marqueurs d'intensité dans l'énoncé discursif laisse à voir l'image de soi ou l'éthos des personnages du roman tels qu'ils sont conçus par le locuteur. Dans le texte 1 de notre corpus, en introduisant dans l'énoncé de Marie, qui est en train de se décrire en comparaison avec les femmes kabyles d'IghilNezman, les atténuateurs « un peu »dans « je me sens un peu comme vos femmes » ${ }^{(21)}$, et « encore moins » dans « elles sont ignorantes, mais j'en sais encore moins qu'elles » ${ }^{(2)}$ l'auteur montre un éthos douteux et hésitant. Marie, l'étrangère au pays de l'autre, s'efforce de se convertir à la kabylie. Elle apprend le kabyle, elle aime sa belle-mère et lui obéit. Elle cherche à maîtriser la cuisine kabyle. Elle ne renonce pas à cette naturalisation kabyle en vue de s'adapter à sa nouvelle vie. Mouloud Feraoun prêche son principe du «vivreensemble » des algériens et des français dans son roman. Mais comme ce n'est pas une affaire facile, Marie hésite entre rester à Ighil-Nezman et retourner chez elle en France, d'où 
l'emploi des atténuateurs dans sa comparaison aux femmes kabyles du village. C'est qu'elle n'est pas tout à fait comme celles-ci. Marie en se voyant «un peu»comme les femmes kabyles atténue l'idée de la ressemblance entre elle et ces dernières. Elle se corrige alors pour ne pas se laisser apparaître hautaine aux yeux de son mari. Alors, elle se décrit comme étant «encore moins» que les femmes du village IghilNezman : «elles sont ignorantes, mais j'en sais encore moins qu'elles ${ }^{(23)}$. L'éthos de Marie laisse voir un moi qui hésite entre un refus d'être au même stade que les villageoises kabyles et et une peur de paraître les mépriser. Marie révèle qu'elle «(se) trouve si heureuse depuis une semaine.» ${ }^{(24)}$. L'emploi de l'amplificateur «si » dans «si heureuse » souligne son désir de mettre en relief un bonheur ressenti de se trouver au sein de la famille d'Amer à Ighil-Nezman. L'emploi du marqueur d'intensité «très bien » dans «Tu sais très bien tout ce que je gagne à être parmi vous. » ${ }^{(25)}$ souligne la volonté de Marie de persuader Amer de sa réelle satisfaction au sein de la famille de son époux. Elle veut dissiper tout doute angoissant l'âme de son mari à propos de leur séjour lui et sa femme au village d'Ighil-Nezman. L'auteur retrace les traits de l'éthos de l'épouse française qui réussit de vivre avec l'Autre kabyle. Elle symbolise le procès de Feraoun selon lequel il s'agit de vaincre la violence et les rébellions, les Français et les Algériens doivent apprendre à vivre ensemble. Le moi et l'autre doivent s'adapter à mener une vie paisible loin des guerres et des révolutions. L'éthos de Marie est amoureux de 
paix et de tranquillité. C'est un éthos qui ne revendique pas la différence ni le racisme. Il cherche à vivre tranquille « et nous serons ici plus tranquilles qu'ailleurs. ${ }^{(26)}$. L'emploi de l'amplificateur «plus » dans «plus tranquilles » est à souligner, il marque le fort désir de Marie de vivre en Algérie et de ne point retourner en France.

Dans le texte (2) de notre corpus discursif, La mère Kamouma s'adresse à Chahba en employant des marqueurs d'intensité qui servent à amplifier la certitude et le degré de puissance de son énoncé qui décrit l'éthos de la femme de Slimane : «Toi, ma fille, tu es plus difficile qu'on ne croit. ${ }^{(27)}$. L'emploi de l'amplificateur « plus» dans «plus difficile qu'on ne croit» met en relief le malice de Chahba. Cette dernière est amoureuse d'Amer, elle éprouve une jalousie à l'égard de Marie. Chahba, malicieuse, essaye d'inviter Kamouma, la mère d'Amer, pour dormir chez elle pour que les deux époux soient à l'aise. Chahba veut d'une part montrer à Marie l'amitié et l'intimité qui se trouvent entre elle et Kamouma la belle-mère de l'épouse française. Et d'une autre, elle n'accepte pas de bon coeur la présence de sa belle mère chez elle. Ce qui pousse Kamouma à décrire Chahba comme étant une femme plus difficile qu'on ne croit. A son tour, Chahba dessine les traits de l'éthos de la mère Kamouma en la décrivant comme étant une femme têtue : «Vous, les Ait-Hamouche, vous avez tous la tête dure. ${ }^{(28)}$. L'emploi de l'amplificateur «tous» dans « vous avez tous la tête dure » est significatif, d'une part, il souligne l'obstination 
de la mère Kamouma, d'une autre, il met en relief la karouba, selon les termes de Feraoun, ou les traits qui unissent les membres d'une même famille. Chahba par l'usage de l'amplificateur «tous» appelle la karouba et rappelle Kamouma de son appartenance aux Ait-Hamouche, aux liens du sang qui les unissent et aux traits de caractère qu'ils partagent.

Dans le texte (3) de notre corpus, se dessinent les éthos de Ramdan, le père de Chahba et d'Amer à travers l'emploi des modalisateurs d'intensité dans les énoncés des deux héros. Ce sont des indices qui marquent la présence des deux héros par un commentaire. Ramdan est l'anneau de connexion entre Amer et sa karouba. Il cherche à maintenir les liens de sang entre Amer et son oncle Slimane. Il défend tantôt Amer devant Slimane, tantôt Slimane devant son neveu. Il accompagne Amer, qui veut rompre avec l'expérience ratée de l'exil, au cimetière d'Ighil-Nezman qui incarne la terre et le sang : La terre car il symbolise le monument qui incarne l'histoire des ancêtres, et le sang incarne karouba et la famille ; on pourrait alors voir les tombes de chaque famille kabyle du village placées ensemble. On peut distinguer facilement les membres défunts de chaque famille regroupés les uns à côté des autres, les liens du sang sont maintenus et respectés même après la mort et l'enterrement. Ramadan se montre nostalgique lors de la visite du cimetière en compagnie d'Amer. Ceci est bien clair dans l'emploi de l'amplificateur «forcément» dans «forcément. Il s'agrandit petit à petit, 
comme le village.» où il compare le cimetière d'IghilNezman qui s'agrandit petit à petit comme le village. Les deux lieux représentent les deux faces d'Ighil-Nezman : les vivants et les morts. Les deux incarnent les liens du sang. Il connait toutes les tombes, l'emploi de l'amplificateur «toutes» dans «Amer: Tu les connais toutes, je parie. » ${ }^{(29)}$ fait paraitre un éthos de Ramdan, le kabyle éprouvant tout signe de reconnaissance et de nostalgie vis-à-vis de sa kabylie. Ramdan incarne l'éthos du héros qui assume sa kabylie et en est fière. Dans l'emploi des amplificateurs «tous, tout» dans «Nous avions tous compris, là-bas. Et de retour au pays nous avons parlé aux Ait-Hamouche. Ils ont tout sang a coulé ! ${ }^{(30)}$, Ramdan souligne la solidarité kabyle. Ils s'unissent et agissent collectivement, même dans leur attitude et leur prise de position à l'égard d'Amer après la mort de Rabah, ils étaient tous solidaires. Tous les Ait-Hamouche décident de se venger d'Amer contre qui ils expriment toute rancune et haine.

De son côté, Ramdan cherche sérieusement à maintenir les liens de sang entre Slimane et Amer d'où l'emploi des amplificateurs «bien sûr, bien » dans «C'est un triste sort, bien sûr. Je sais bien, mon pauvre garçon, que tu n'y es pour rien ${ }^{(31)}$, Ramdan joue le rôle du réconciliateur qui s'efforce de rapprocher les deux personnages en conflit «Nous ne voulons que votre bien, à Slimane et à toi. Inutile de sourire, tu n'es plus un enfant : je te parle franchement» L'usage de l'amplificateur «franchement» intensifie le degré de 
puissance de l'énoncé de Ramdan qui veut persuader Amer de sa volonté de réparer cette rupture entre lui et son oncle.

Amer est sûr qu'il reconnaît toutes les familles kabyles du village, il veut se débarrasser du poids de la honte qui le tourmentait tout au long de son exil. Le degré de puissance du témoignage d'Amer est atténué par l'emploi du modalisateur d'intensité «bien sûr » dans «Bien sûr que je les reconnais ». De retour à son village natal, Amer éprouve de l'ambarras à l'égard de ses parents obligés de vendre leurs terrains pour vivre. Le chemin vers le cimetière est un chemin vers la quête de son identité et sa dignité perdues dans son exil. L'usage de l'atténuateur «quelques» dans «Je n'ai pas besoin que de quelques précisions » met en relief le désir d'Amer de se montrer conscient des moindres détails de son histoire, l'histoire des origines kabyles et de ses ancêtres. Il n'a besoin que de quelques précisions pour reconnaitre tous les coins du cimetière d'Ighil-Nezman. L'usage des modalisateurs d'intensité dans les énoncés d'Amer montre un éthos nostalgique, trop attaché à sa kabylie et aspirant à reconquérir son identité natale.

3. La dialectique de l'usage de la personne dans le discours identitaire

C'est dans et par le langage que l'homme se constitue comme sujet; parce que le langage seul fonde en réalité, dans sa réalité qui est celle de l'être, le concept d' « ego » ${ }^{(32)}$

Est « ego » qui dit « ego » ${ }^{(33)}$ 
Dans une scène énonciative, la communication implique un locuteur, c'est le «je » ou l' « ego » et un interlocuteur, le «tu», qui sont les deux bouts de la communication. Selon Benveniste, c'est "par le langage que l'homme se constitue comme sujet; parce que le langage seul fonde dans sa réalité qui est celle de l'être, le concept d' «ego». C'est par le langage qu'un locuteur $\mathrm{A}$ se définit en s'opposant à un interlocuteur $(\mathrm{B})$. En soumettant notre corpus à une analyse quantitative, nous notons les deux déictiques personnels Je et tu significatifs car la fréquence de leur apparition est nettement supérieure à la moyenne. Benveniste dénote que le fondement de la subjectivité se détermine par le statut linguistique de la personne. « La manifestation de la subjectivité ne prend relief qu'à la 1ère personne. » ${ }^{(34)}$

Figure 4 : La fréquence de la personne dans le texte 1

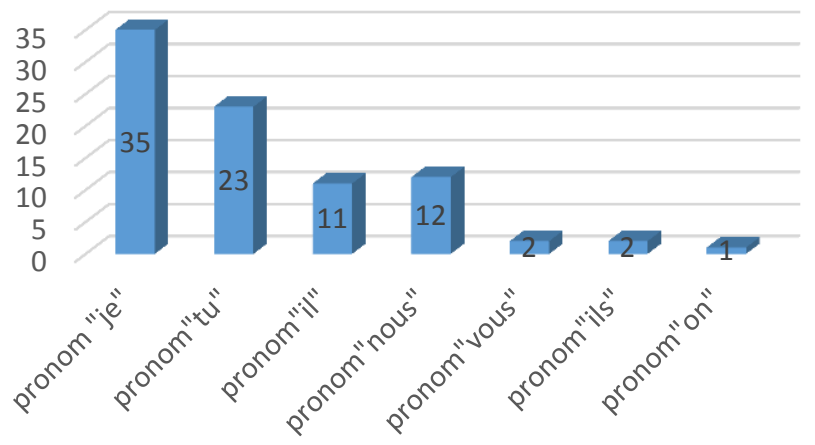

Figure 5 : La fréquence de la personne dans le texte 2 


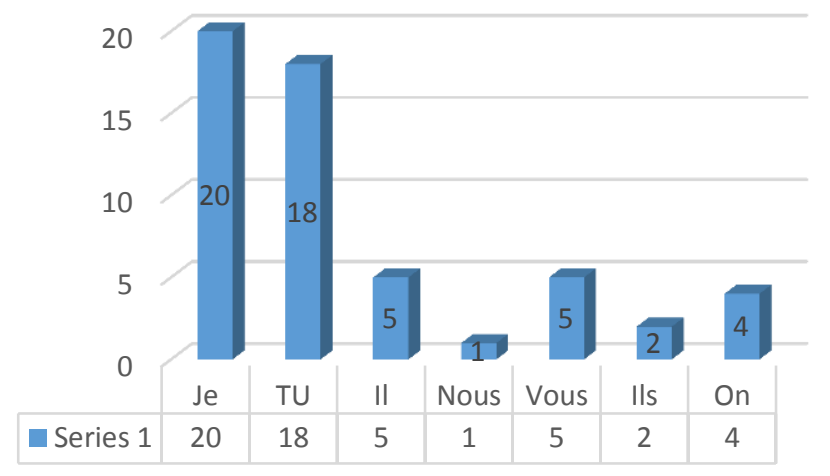

Figure 5 : La fréquence de la personne dans le texte 3

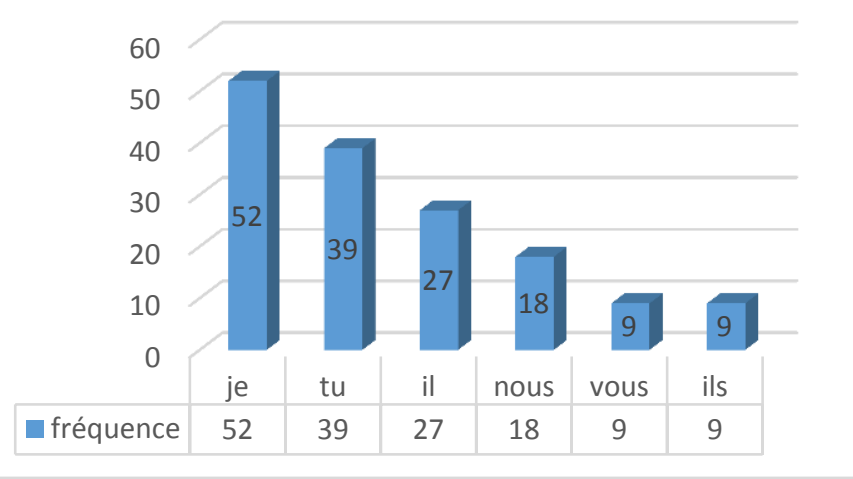

Le Je dans tout discours, dans tout acte allocutionnaire cherche à influencer son destinataire, d'agir sur lui, car énoncer c'est orienter vers une certaine visée, c'est influencer. E. Benveniste définit le discours comme étant «toute énonciation supposant un locuteur et un auditeur, et chez le premier l'intention d'influencer l'autre en quelque manière. ${ }^{(35)}$. Le Je discursif tend à projeter une image de soi à travers la scène énonciative. C'est le Je de l'énonciateur, le producteur physique de l'énoncé, c'est l'instance interne de 
l'énonciation. Ce Je projette aussi l'image du locuteur, l'instance énonciative externe et qui est le producteur effectif de l'énoncé. Quels éthos Mouloud Feraoun cherche-t-il à projeter à travers le Je discursif de son énonciation? Et quelle image de soi tient-il à émettre à travers le Je de l'énonciateur de son texte discursif?

L'auteur, natif de Kabylie, met en relief le procès de l'émigration kabyle vers la France pour fuir la misère du peuple algérien colonisé. Feraoun était lui-même témoin de cette misère qui l'a profondément marqué. Amer, protagoniste du roman, incarne l'image du kabyle qui retourne à ses origines en quête de son identité perdue pendant une dizaine d'années d'exil.

Dans le texte (1) de notre corpus, Le «je » d'Amer s'adresse à un «tu» de Marie dans ce que Benveniste nomme le cadre figuratif ${ }^{(36)}$. Qui est Amer pour son interlocuteur, quelle image Amer est-il en train de se forger aux yeux de son interlocuteur et quelle identité Amer donne-t-il de luimême.

«Amer : A Paris, j'avais besoin de me sentir aimé. Et je n'avais que toi. Je sais ce que c'est d'être étranger, c'est un état pitoyable pour l'homme. » ${ }^{37}$

Dans le dialogue entre Amer et Marie, le Je du locuteur ne peut se présenter et advenir en tant que sujet que dans son rapport à l'autre. Le ««Je» implique automatiquement un « $\mathrm{Tu}$ »: c'est dans cette relation constitutive qu'émerge une subjectivité qui désigne aussi une identité. ${ }^{(38)}$. L'éthos 
d'Amer se présente dans son rapport à Marie, et réciproquement l'image du moi de Marie ne se dévoile que dans le cadre de la relation qui l'unit à son allocutaire Amer.

Dans cet extrait du texte (1), le Je d'Amer dessine les traits d'un éthos malheureux, vaincu par le déracinement ressenti en France. C'est le statut de tout émigré, c'est le sentiment de l'étranger dans le pays de l'inconnu. Il a besoin d'être aimé dans une société où il ressent la différence. A Paris, Amer avait besoin d'être aimé, il connaît bien ce que c'est être étranger. L'usage du verbe de l'opinion savoir dans « je sais ce que c'est d'être étranger» marque l'adhésion du locuteur à son énoncé, ce qui augmente la certitude et la puissance de l'énoncé. Le qualificatif «pitoyable» employé après le syntagme nominal «un état »est à connotation négative, il joue sur l'émotion de l'allocutaire.

Julia kristeva définit l'étranger comme étant :

(...) l'étranger (qui) nous habite : il est la face cachée de notre identité, l'espace qui ruine notre demeure, le temps où s'abîment l'entente et la sympathie. De le reconnaitre en nous, nous nous épargnons de le détester en lui-même. Symptôme qui rend précisément le «nous»problématique, peut-être impossible, l'étranger commence lorsque surgit la conscience de ma différence et s'achève lorsque nous nous reconnaissons tous étrangers, rebelles aux liens et aux communautés. ${ }^{(39)}$

Ce sentiment de l'étranger est mis en relief à travers l'usage du déitique dans le texte discursif (2) où les trois 
héroïnes féminines du roman de Feraoun se sentent étrangères l'une par rapport à l'autre. Rare est l'usage du déictique «nous» dans le texte (2) ( une seule fois mettez la citation) en comparaison avec le texte 1 où la première personne du pluriel est utilisé une seule fois à comparer au texte (1) où le «je » et le «tu » s'unissent en «nous», signe d'un grand désir de la part du couple de franchir les barrières qui se dressent entre eux, ils rêvent de surmonter les difficultés qui les séparent. Au contraire, Kamouma, Chahba et Marie, les hérö̈nes du texte 2, sont tout à fait différentes l'une de l'autre. Quant à Marie, la jeune française, elle s'efforce de se conformer à la vie kabyle tout en luttant contre sa condition d'étrangère et de s'adapter au sein d'un milieu qui lui est différente. Mais souvent elle échoue à suivre le discours berbère de Chahba et sa belle-mère. Elle exprime à plusieurs reprises son échec à les comprendre toutes deux, et comment le faire avec deux femmes qui ne cessent pas de jouer sur l'implicite et attaquer la jeune française sans que cette dernière ne le sache. Le lecteur de ce discours éprouve de la pitié envers Marie qui plonge dans l'incompréhension et le malentendu.

Marie : Achou! dit Madame.

Chahba :Celle-là ! je m'en vais lui expliquer; moi. Voilà Madame : nana Kamouma, couchée maison Chahba. ${ }^{(40)}$

Dans ce texte, se montrent la dureté et la violence de se trouver en contact avec des personnes dont on ne comprend pas la langue. La barrière de la langue a causé pas mal de 
malentendus dans le dialogue entre Marie d'un côté et Chahba et Kamouma d'un autre. Chahba désigne Marie par «Madame» ce qui élargit la mise à distance entre la jeune française et son entourage. Dans cet extrait du texte, Chahba ne tutoie pas Marie, elle la désigne par le pronom personnel complément d'objet «lui », et le démonstratif «celle-là», signes d'indifférence à l'égard de Marie dont la jeune berbère est jalouse.

Chahba : C'est cela. On te laisse, ton gros bête. D'ailleurs tu le mérites bien. N'est-ce pas, nana, qu'elle est belle, ta bru? ${ }^{(41)}$

Chahba se moque de Marie, elle ironise Amer en le désignant par «Gros bête ». «On te laisse, ton gros bête » est un énoncé à valeur péjorative où le pronom personnel « on » a la valeur de nous (Chahba + kamouma) qui s'unissent en un un lorsqu'il s'agit de dévaloriser l'épouse française. Kamouma n'aime pas Marie qui est à ses yeux l'étrangère qui partage avec elle l'amour de son fils et qui la menace de le lui priver à jamais si elle prend la décision de retourner en France. En plus, Marie n'est pas encore enceinte, ceci inquiète la mère Kamouma qui rêve de voir ses petits-fils avant de quitter la vie. Perpétuer l'héritage pour que la terre soit toujours propriété de la famille est l'objectif de toute mère kabyle qui n'hésite pas à s'immiscer dans la vie de ses fils en vue de les remarier à d'autres femmes si les leurs sont stériles.

Kamouma : Bien, bien. Je demanderai à mon fils. Tu sais, jusqu'ici, je ne les ai pas gênés là, dans ma soupente. Je tombe 
endormie chaque soir comme une souche. Et je ne m'occupe pas d'eux. Ils parlent à leur aise. Je n'y comprends rien. Pour le reste... ${ }^{(42)}$

Kamouma, comme Chahba, désigne le couple par le pronom personnel troisième personne de pluriel dans «ils parlent à l'aise», et par le pronom personnel complément d'objet troisième personne du pluriel «eux» dans «je ne les ai pas gênés là, dans ma soupente » et «Et je ne m'occupe pas d'eux ». Kamouma est tout à fait indifférente à l'égard de sa belle-fille, elle ne lui adresse pas la parole. Elle projette à travers son discours une image de soi, c'est l'image stéréotypée de la mère berbère qui fière de ses origines, aurait bien aimé que son fils se marie d'une berbère et non d'une française, donc étrangère. Cela explique la rancune que Kamouma éprouve à l'égard de sa belle-fille. Cette rancune se traduit en une complète indifférence à l'égard de la jeune épouse.

Chahba laisse apparaitre à travers sa parole l'ethos de la jeune berbère qui n'hésite point à défendre son amour, elle s'engage et avec violence dans une lutte en vue de s'accaparer de La mère Kamouma, de s'approcher d'elle et de la gagner de son côté. Chahba, la belle brune qui attire par sa beauté et son charme Amer qui lui à son tour ne risque point de lui révéler sa passion. Elle représente l'image de l'intelligente berbère qui lutte pour son amour. Sa décision de rapprocher les deux familles : Les Ait-Larbi et les Ait-Hamouche en conflit depuis longtemps témoigne de son intelligence. 
Dans le texte (3), Ramdan, le père de Chahba, déploie de grands efforts en vue de remédier la blessure de sang qui a rompu les liens entre les deux grandes familles d'IghilNezman. L'usage du pronom personnel sujet «nous » révèle ce désir éprouvé de la part de Ramdan d'unir les deux membres en conflit : Slimane et Amer. Nous notons ainsi la fréquence de l'emploi de déitique «nous» et de ses variantes; « notre » dans ce passage.

Ramdan : Le vrai village, ce n'est pas celui qui se dresse fièrement sur la crête. C'est celui-ci : figé dans notre terre, immobile et éternel mais peu effrayant à mon avis, parce que nous le connaissons bien, nous les vivants. Nous nous habituons chacun à notre place et nous n'avons pas peur d'y venir. ${ }^{(43)}$

Ramdan se dévoile à travers sa parole, il laisse apparaitre un éthos qui découle du dit, selon les termes d'Amossy ${ }^{(44)}$. Ramdan représente l'image du sage berbère qui croit au concept du vivre-ensemble et du pardon malgré la haine et le sang. Slimane ne pouvait point oublier que son neveu Amer a été la cause du meurtre de son frère Rabah en France. Mais Ramdan qui incarne le stéréotype du vieux sage berbère dont l'expérience et la bonté donnent à son discours plus de poids, d'efficacité, a le pouvoir d'agir sur son interlocuteur. Tout lui obéit et a confiance en lui. Lui seul est capable de réconcilier les deux familles et mettre un terme à leurs hostilités. Ramdan emploie le déictique personnel de premiere «nous» sept fois dans l'extrait ci-dessus, ce qui connote son fort désir 
de sauver son village de cette division au sein d'IghilNezman. Ramdan projette l'éthos d'une âme nostalgique voulant mettre en relief ses origines kabyles L'auteur Feraoun est présent partout, il est cette voix qui domine le texte, ce sujet qui est au-delà du texte à travers lequel il agit sur le lecteur. Il projette de soi-même dans le texte. L'image qu'il projette de soi-même ${ }^{(44)}$ est l'ethos d'une âme nostalgique voulant mettre en relief ses origines kabyles.

«Notre terre est modeste. Elle aime et paie en secret. Elle reconnait tout de suite les siens : ceux qui sont faits pour elle et pour qui elle est faite.» ${ }^{(45)}$

«(..) notre terre aime les fellahs rudes et avares. »(46)

Le texte de la non-personne de Feraoun permet d'interpréter l'embrayeur «nous », c'est la personne par laquelle l'auteur du roman se désigne. Ce nous qui domine dans le texte englobe en premier lieu la forte présence de l'auteur mais aussi nous pouvons y déceler un vous du lecteur.

Nous=je de l'auteur en prédominance + ils les membres tribus kabyles + vous lecteurs

Conclusion :

Toute parole vient d'un énonciateur incarné; même écrit, un texte est porté par une voix, celle d'un sujet au-delà du texte. $^{(47)}$ (Maingueneau, 2000 : p.77)

Se distancier du narré est une stratégie de l'auteur qui adopte un mode impersonnel dans la narration du texte romanesque de Feraoun, mais sa subjectivité ne tarde pas à apparaitre. L'auteur a créé tout un dédoublement énonciatif, un 
dialogisme intérieur, des jeux d'implicite à l'aide d'un usage maîtrisé des modalisateurs discursifs. La fréquence de l'emploi des modalisateurs de négation a reflété une polyphonie énonciative qui témoigne l'hybridité de l'ethos du héros du roman de Feraoun. C'est un héros en pleine contradiction et dont l'âme témoigne de la lutte existentielle entre le moi et l'autre. L'occurrence de la négation dans le discours du roman de Feraoun fait preuve de cette lutte identitaire où son héros s'engage. La fréquence d'emploi de cet outil linguistique de modalisation est connotative et laisse tant voir de l'ethos de l'auteur qui est lui-même en lutte entre son moi kabyle et l'autre français.

L'auteur s'est déclaré toujours présent dans le discours des héros de son roman à l'aide de l'emploi des adoucisseurs et des amplificateurs marquant tantôt une certitude, tantôt un doute à l'égard du contenu propositionnel de son énoncé. Le lecteur détecte la présence et l'implication de Feraoun dans le discours de son roman grâce à cette variation de degré de puissance de l'énoncé ; cette stratégie a aidé l'auteur à projeter l'image de soi à travers le discours de ses héros, de se présenter et enfin de subjectiviser son récit impersonnel. 


\section{Notes:}

1-Otten, M, Méthodes du texte, introduction aux études littéraires, Editions Duclot, Paris Gembloux, 1987. p.342.

2- Maingueneau, Dominique, Analyser les textes de communication, Paris, Nathan, 2000, p.81

3-Amossy, Ruth, La présentation de soi : ethos et identité verbale, paris, Presse universitaire de France, 2010, p. 45

4-Amossy, Ruth, L'argumentation dans le discours, Paris, NathanUniversité,2000, p.219

5-Feraoun, Mouloud, La terre et le sang, Paris, Le Seuil, 1953, p. 49.

* Notre corpus se constitue de trois extraits du texte discursif du roman La terre et le sang de Mouloud Feraoun : texte (1) p.p. 49-50, texte (2) p.p.123-124, texte (3) p.p. 113-120.

\section{6- Ibid}

7-O. Ducrot, Le dire et le dit, Paris, Minuit, 1984, p. 215.

8-Ibid pp. 216,217

9-« le sens de l'énoncé, dans la représentation qu'il donne de l'énonciation, peut y faire apparaitre des voix qui ne sont pas celles d'un locuteur. J'appelle «énonciateurs» ces êtres qui sont censés s'exprimer à travers l'énonciation, sans que pour autant on leur attribue des mots précis ; s'ils parlent, c'est seulement en ce sens que l'énonciation est vue comme exprimant leur point de vue, leur position, leur attitude, mais non pas, au sens matériel du terme, leurs paroles »Ibid, p.204

10-J. Moeschler, Les aspects pragmatiques de la négation linguistique: acte de langage, argumentation et inférence pragmatique, Travaux du Centre de Recherches Sémiologiques, nº 59, Neuchâtel 1991, p. 124. 11-Feraoun, p. 8

12-«Marie: Ce n'est pas pour te le rappeler que j'en parle. Au contraire, je me trouve si heureuse depuis une semaine! Tu sais très bien tout ce que je gagne à être parmi vous ». Ibid, p.49

13-Ibid. 
14-« Amer : Aussi, mais peut-être moins. Du moment qu'elle a son homme qui tient à elle, elle n'est plus étrangère. Elle entre dans la famille. ». Ibid, p.49

15-Ibid.

16-« Marie: Je n'ai pas grand-chose à regretter. Pour le reste, bien entendu, j'avais confiance en toi. Sinon autant rester là-bas. Mais ne te figure pas que je vais abuser de la situation et devenir exigeante. Si tu m'en crois, ne nous faisons pas trop d'idées. Nous vivrons simplement et nous serons ici plus tranquilles qu'ailleurs. Voilà. Et trêve de discours ! Je vois sur le chemin trois femmes qu'il me faut rattraper. Et allons voir chez nous ce que fait maman... Comment dites-vous? ». Ibid, p.50.

17-Ibid.

18-«Kamouma : Bien, bien. Je demanderai à mon fils. Tu sais, jusqu'ici, je ne les ai pas gênés là, dans ma soupente. Je tombe endormie chaque soir comme une souche. Et je ne m'occupe pas d'eux. Ils parlent à leur aise. Je n'y comprends rien. Pour le reste... ». Ibid, p.123

19-«Amer : Père Ramdan, je ne leur demande pas de comptes. Est-ce qu'ils t'ont chargé de me demander ? C'est mon retour qui vous gêne. Tu voudrais que je m'offre à votre gendre ou que j'aille au diable. C'est ça? La mort de rabah m'a longtemps obsédé. Crois-tu que j'oublie et que j'aie besoin de tes évocations? Je sais que je ne suis pas coupable. Je n'ai peur ni de Slimane, ni d'autre ! Ibid, p.118.

20-Dictionnaire de la linguistique, Jean Dubois et al, 2002

21-« Marie: Veux-tu que je te dise? Eh bien, moi, depuis que je suis ici, je me sens un peu comme vos femmes : elles sont ignorantes, mais j'en sais encore moins qu'elles. En attendant de bien comprendre, je me laisse aller. Si, un jour ou l'autre, ça ne marche plus, nous nous en retournerons. C'est tout. ». Feraoun, p.49.

22-Ibid.

23-Ibid. 
24-« Marie: Ce n'est pas pour te le rappeler que j'en parle. Au contraire, je me trouve si heureuse depuis une semaine! Tu sais très bien tout ce que je gagne à être parmi vous. »Ibid, p.49.

\section{5-Ibid.}

26-«Marie: Je n'ai pas grand-chose à regretter. Pour le reste, bien entendu, j'avais confiance en toi. Sinon autant rester là-bas. Mais ne te figure pas que je vais abuser de la situation et devenir exigeante. Si tu m'en crois, ne nous faisons pas trop d'idées. Nous vivrons simplement et nous serons ici plus tranquilles qu'ailleurs. Voilà. Et trêve de discours ! Je vois sur le chemin trois femmes qu'il me faut rattraper. Et allons voir chez nous ce que fait maman... Comment dites-vous? ». Ibid, p.50.

27-Ibid, p.123.

28-Ibid.

29-Ibid, p.116

30- Ibid, p.118

31- Ibid, pp.117-118

32-Benveniste, Emile, Problèmes de linguistique générale,Tome I, Paris, Gallimard, 1966, p.259

33-Ibid, p. 260

34-Ibid, p. 264

35-E. Benveniste, problèmes de linguistique générale 2, Gallimard, 1974, p.241,242

36-Selon Benveniste, l'énonciation « pose deux figures également nécessaires, l'une source, l'autre but de l'énonciation »Ibid, p. 82

37-Feraoun, p.49

38-Amossy, $2010: 105$

39-Kristeva, Julia, Étrangers à nous-mêmes, Paris, Gallimard, 1988, p.9

40-Feraoun, 123, 124

41-Ibid, p.124

42- Ibid, p.123

43- Ibid, p.117 
44-«En effet, l'image de soi peut découler du dit : ce que le locuteur énonce explicitement sur lui-même en se prenant comme thème de son propre discours. En même temps, elle est toujours un résultat du dire : le locuteur se dévoile dans les modalités de sa parole, même lorsqu'il ne se réfere pas à lui-même. » In Amossy, Ruth, La présentation de soi : ethos et identité verbale, Paris, Presse universitaire de France, 2010, p.113

45- Feraoun, p.162

46- Ibid, p.163

47- Maingueneau, , $2000: 77$

Corpus :

Feraoun, Mouloud. 1953. La terre et le sang, Le Seuil. Paris.

Références bibliographiques :

1-Amossy, Ruth. 2000. L'argumentation dans le discours. NathanUniversité. Paris.

2-Amossy, Ruth. 2010. La présentation de soi : ethos et identité verbale. Presse universitaire de France. Paris.

3- Benveniste, Emile. 1974. Problèmes de linguistique générale, tome II, Gallimard. Paris.

4- Benveniste, Emile. 1996. Problèmes de linguistique générale, tome I. Gallimard. Paris.

5-Ducrot, Oswald et Tzvetan Todorov. 1972. Dictionnaire encyclopédique des sciences du langage. Éditions Du Seuil.

6-Ducrot, Oswald. 1984. Le dire et le dit. Éditions Minuit. Paris. 7-

8-Dubois, Jean. Et al. Dictionnaire de la linguistique. 2002. LarousseBordas/VUEF. Paris.

9-Maingueneau, Dominique. 2000. Analyser les textes de communication, Nathan, Paris.

10-Otten, M. 1987. Méthodes du texte, introduction aux études littéraires. Editions Duclot. Paris Gembloux. 
11-Moeschler, J. 1991. Les aspects pragmatiques de la négation linguistique : acte de langage, argumentation et inférence pragmatique, Travaux du Centre de Recherches Sémiologiques, $\mathrm{n}^{\circ}$ 59, Neuchâtel.

12-Vion, Robert. 2012. La modalisation: un mode paradoxal de prise en charge, in Filol. linguíst. port.,n. 14(2), p.203-224. 